\title{
Correlations between Glycosylated Hemoglobin and Lipid Profiles in Newly- Diagnosed Type II Diabetics
}

\section{Rao $\mathrm{SN}^{1^{*}}$, Kuldeep $\mathrm{GB}^{2}$}

${ }^{1}$ Department of Biochemistry, AECS Maruthi Dental College, Bengaluru, Karnataka, India; ${ }^{2}$ Department of Medicine, Sri Krishna Sevashrama Hospital, Bengaluru, Karnataka, India

\begin{abstract}
Introduction: Type II diabetes is a chronic disease which results from aspects such as complex inheritance interaction, obesity, and sedentary lifestyle. In India, diabetes is turning into an epidemic as currently, more than 62 million individuals suffer from the disease. To our knowledge, very few studies have evaluated the correlation between lipid profiles and glycated hemoglobin (HbA1c) in newly diagnosed type II diabetes patients with hypertension. The early detection of lipid abnormalities in these patients will help prevent the cardiovascular outcomes.

Objectives: To identify patterns of dyslipidemia among newly diagnosed type II diabetes mellitus (DM) patients with and without hypertension in Bengaluru (urban and rural) in Karnataka (South India); and to identify correlations between HbA1c levels and lipid profiles.

Methods: This was a cross sectional study involving 194 individuals in Bengaluru, India from the period of April to December 2017. Demographics, lifestyle habits and clinical features were analyzed for the presence of any interrelationship with the occurrence of diabetic dyslipidemia. One-way analysis of variance (ANOVA), followed by Tukey's post hoc tests, Chi square and correlation studies were used to establish a significant level of association between the study parameters.

Results: Among non-diabetics, prediabetics, diabetics and diabetics with hypertension, there were significant differences in lipid profiles, as well as levels total cholesterol, triglycerides, high density lipoprotein (HDL), low density lipoprotein (LDL), very low-density lipoprotein, ratios of cholesterol to HDL and ratios of LDL to HDL. Positive correlations were observed between HbA1c and fasting blood sugar (FBS), and random blood sugar (RBS) in non-diabetics; whereas, in prediabetics, the RBS highly correlated with HbA1c and negatively correlated with HDL. In diabetics, both fasting and random blood sugar highly correlated with $\mathrm{HbA1c}$, however, no significant correlation was observed between HbA1c and any of the tested lipid profiles in non-diabetics and diabetics. A strong correlation between $\mathrm{HbA1c}$ and lipid profiles was established.

Conclusion: An overweight diabetic man with poor glycaemic control, over the age of 46 years, having a desk job or working as a driver or businessman, with abdominal obesity, leading a sedentary lifestyle and having habits such as alcohol drinking and smoking is at high risk for developing hypertension.
\end{abstract}

Keywords: Diabetes; Diabetic dyslipidemia; Hypertension; Glycosylated haemoglobin

*Correspondence to: Sujatha Narayana Rao, Biochemistry Department, AECS Maruthi Dental College, Off Bannerghatta Road, Bengaluru, India 560076, Tel: +91 9916626524; E-mail: sujatharao.8@gmail.com

Received: August 19, 2019; Accepted: October 17, 2019; Published: October 24, 2019

Citation: Rao SN, Kuldeep GB (2019) Correlations between Glycosylated Hemoglobin and Lipid Profiles in Newly-Diagnosed Type II Diabetics. J Diabetes Metab 10:836. doi: 10.35248/2155-6156.19.10.836

Copyright: (C) 2019 Rao SN, et al. This is an open-access article distributed under the terms of the Creative Commons Attribution License, which permits unrestricted use, distribution, and reproduction in any medium, provided the original author and source are credited. 


\section{INTRODUCTION}

Diabetes mellitus (DM), characterized by hyperglycemia, constitutes of a 'group of chronic, hereditary, metabolic disorders' affecting millions of people worldwide each year [1,2]. It complicated by nephropathy, dyslipidemia, retinopathy, cardiac and or cerebrovascular disease, and organ failure. There is a high rate of mortality among diabetics.

Determination of hyperglycemia is performed using random blood sugar (RBG), fasting blood sugar (FBG) and glycosylated hemoglobin (HbA1c). Of these, HbA1c is considered the standard routine indicator of glycemic control [3,4]. Moreover, $\mathrm{HbA1c}$ may forecast risks of the advancement of diabetic-related complications [2]. One of the most common complications is abnormal levels of serum lipids, also called dyslipidemia [1]. Many studies have attempted to identify patterns of dyslipidemia and their correlation with HbA1c. However, the results have been contradictory [5]. While some studies have shown that all parameters of lipid profile of diabetics correlate with $\mathrm{HbA1c}$ [6-8] others found no such correlation [9-11]. Hussain et al. [8] found a direct correlation between $\mathrm{HbA} 1 \mathrm{c}$ and triglycerides and an inverse correlation between $\mathrm{HbA1c}$ and LDL in Afghani diabetics. Similarly, Khan [12] showed a direct correlation between HbA1c and LDL and TC in diabetics in Saudi Arabia. Maharjan et al. [13] showed that $\mathrm{HbA1c}$ was a predictor of dyslipidemia among type 2 diabetics. The predictive value of $\mathrm{HbA1c}$ varies among races and ethnicities because the rate of glycation and/or RBC life span differs among racial and ethnic groups [14,15]. There is also influence of genetic factors on HbA1c levels as shown by Snieder [16] in a study of healthy and diabetic populations. The previous contradictory findings, the fact that HbA1c levels are genetically determined and that their levels as a predicting factor differs among races and ethnicity, inspired us to undertake this study because is the diabetes capital of the world [17]. As such, it is critically essential to explore the possibility of a relationship of glycosylated hemoglobin and lipid profiles of individuals in the current context for early detection and timely action to reduce economic burdens associated with diabetes [11]. Moreover, our study is important because of the enormous numbers of people afflicted with diabetes and those likely to become diabetic. Our study aims to evaluate patterns of dyslipidemia among newly diagnosed type II DM patients with and without hypertension in Bengaluru (urban and rural) in Karnataka (South India) and to identify correlations between HbA1c levels and lipid profiles.

\section{MATERIALS AND METHODS}

This is a cross sectional study conducted at the Shree Krishna Sevashrama Hospital, Bengaluru, Karnataka, India from April to December 2017. A total of 194 individuals was included in this study and matched for age and gender. After obtaining the Institutional ethical committee approval, informed consent was taken from all the individuals participated in this study.

The subjects were divided into four groups. The exclusion criteria for all the four groups included pregnant and lactating women. Group I (controls) and Group II (prediabetics) included individuals presenting for the 'Master Health Checkup' plan offered by the hospital. The inclusion criteria were as follows: Group I were 'controls or non-diabetics' with HbA1c equal to or below 5.6. Individuals with $\mathrm{HbA1c}$ in the range of 5.7 to 6.4 were grouped as prediabetics, forming Group II as per WHO criteria. Fifty-four apparently healthy individuals aged 21 to 70 years of either gender were selected as Group I (controls), while only 12 individuals aged 25 to 83 years with $\mathrm{HbA1c}$ in the range of 5.7 to 6.4 were considered Group II (prediabetics). Group III (Type II DM) consisted of seventy-two outpatients aged 31 to 74 years and newly diagnosed with type II diabetes mellitus (as per WHO criteria) with HbA1c 6.5 and above. This group also excluded patients without complications, including hypertension. Group IV (Type II DM + hypertension) included 56 inpatients age 30 to 68 years of both genders with newly diagnosed type II DM and with hypertension. The inclusion criteria for this group were HbA1c 6.5 and above, type II DM and hypertension. Figure 1 demonstrates the flow chart for the selection of study subjects. Informed consent was obtained from each individual. The study was approved by the ethics committee of the institution.

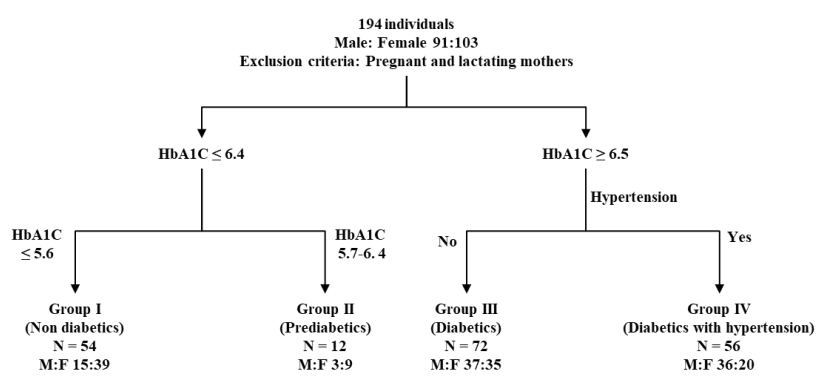

Figure 1: Flow chart for the selection of the study population.

The demographic study variables used for collecting data included age, gender, smoking and drinking behavior. The age and gender of the individuals were obtained from their hospital records. The health examination provided the anthropometric measurements, including weight and height that were used to calculate the body mass index (BMI). Weight was measured using an electronic digital scale, and height was measured using a wall-mounted stadiometer. BMI was calculated as weight $(\mathrm{Kg})$ per height squared $\left(\mathrm{m}^{2}\right)$. BMI was divided into standard four categories as follows: Under normal $\left(<19 \mathrm{Kg} / \mathrm{m}^{2}\right)$, Normal $\left(19-24.9 \mathrm{Kg} / \mathrm{m}^{2}\right)$, Overweight $\left(25-30 \mathrm{Kg} / \mathrm{m}^{2}\right)$ and Obese $(>20$ $\left.\mathrm{Kg} / \mathrm{m}^{2}\right)$. The waist:hip circumference was considered the measure for abdominal obesity and was categorized by its presence (yes) or its absence as (no). Occupation and other lifestyle habits such as food types, alcohol intake, smoking habits and level of physical activity were individually recorded. Food type included vegetarian or non-vegetarian. Consumption of alcohol, recorded as yes, included both present and ex-drinkers. Similarly, smokers were recorded as yes, including individuals who currently smoke those who formerly smoked. The level of physical activity was categorized as sedentary with nil to almost nil physical activity, moderately active with medium levels of physical activity in their daily lives such as walking, and active included individuals who jog and walk.

Blood samples were obtained for biochemical tests. Sample collection involved venous whole blood samples in labeled 
EDTA tubes directly used for the analysis of HbA1c and fasting blood sugar after an overnight fast $(10 \mathrm{~h})$ and a portion was allowed to clot. Serum was separated and used for the analysis of lipid profile (total cholesterol (TC), triglycerides (TG), high density lipoprotein (HDL), low density lipoprotein (LDL), very low-density lipoprotein (VLDL), ratio of cholesterol to HDL, ratio of $\mathrm{LDL}$ to $\mathrm{HDL}$ ). All these were analyzed using an Olympus AV Autoanalyser (using diasys reagents) manufactured by Diasys Diagnostic system GmbH, Holzheim, Germany. Glycosylated hemoglobin ( $\mathrm{HbA1c}$ ) was measured using the particle-enhanced immune turbidimetric method. Glucose was assessed using the enzymatic glucose oxidase (GOD) and peroxidase (POD) method. Lipid components such as total cholesterol were measured using the cholesterol oxidaseperoxidase (CHOD-POD) enzymatic photometric method, while HDL was determined using antihuman $\beta$ lipoprotein antibodies that liberated only HDL-cholesterol that in turn were analyzed by the enzymatic (CHE, CHO, POD) method. LDL was determined using homogeneous whole direct measurement using the color-producing enzymatic reaction as only LDL is selectively protected and then released. TG was calculated using the glycerol 3-phosphate oxidase (GPO) enzymatic method, whereas VLDL was measured by performing an indirect calculation from TG result using the Friedwald formula.

Continuous variables were expressed as mean \pm standard deviation, while categorical data were expressed as frequencies and percentages. Continuous data were converted to categories wherever convenient. A one-way ANOVA was conducted for the continuous variables to compare the means of the populations, followed by Tukey's post hoc tests, and Chi square $\left(\chi^{2}\right)$ tests were used for the categorical variables to evaluate the significance of the study parameters. The statistical difference between the groups was measured by ANOVA and among the groups which group specifically differed was measured by Tukey's post hoc test. In this study, ANOVA along with Tukey's test was chosen because of the unequal sample sizes between the groups. The correlations were measured using Pearson's coefficient of correlation $(\mathrm{r})$ between study variables. The results of all tests with $\mathrm{p}<0.05$ were considered statistically significant. All statistical analyses were performed using the SPSS statistical package version 24 .

\section{RESULTS}

Table 1 illustrates the descriptive statistics and the results of ANOVA for FBS, RBS, HbA1c and the lipid profiles of nondiabetics, prediabetics, diabetics and diabetics with hypertension. There were significant differences among the various populations in terms of all studied variables. There are clear increases in levels of all components except for HDL among the four populations, with minimum levels in nondiabetics and maximum levels in diabetics with hypertension. The range that has been considered to be desirable for FBS is 70-100 and for RBS it is 70-140. The desirable range for TC, TG, LDL, VLDL, TC: HDL and LDL:HDL are less than 200 $\mathrm{mg} / \mathrm{dl}, 150 \mathrm{mg} / \mathrm{dl}, 130 \mathrm{mg} / \mathrm{dl}, 40 \mathrm{mg} / \mathrm{dl}, 4: 1$ and $3.5: 1$ respectively. The desirable level of HDL is greater $40 \mathrm{mg} / \mathrm{dl}$. For FBS, only the non-diabetics were in the desirable range; both the non-diabetics and prediabetics were in the desirable range for RBS. Therefore, FBS can be considered the better indicator of diabetes between the two, because FBS as able to identify even the prediabetics. Within the lipid, the mean of the total cholesterol, LDL and the ratio of LDL: HDL of the diabetics with hypertension was more above desirable levels. The TG and VLDL levels of diabetics and diabetics with hypertension were higher than the desirable range, while those of prediabetics were in the desirable range. The average levels of HDL and the ratio between TC and HDL showed only non-diabetics to be in the desirable range. The glycemic control among the diabetic population was such that the majority $(63.9 \%)$ of diabetics had inadequate control, followed by $19.4 \%$ with poor control and the remainder $(16.7 \%)$ with good control. The glycemic control of the diabetics with hypertension showed mostly poor control with $67.9 \%$, followed by the remainder $(32.1 \%)$ with inadequate control.

Table 1: Descriptive statistics and ANOVA for the study variables.

\begin{tabular}{lccccc}
\hline & \multicolumn{4}{c}{ Population (Mean \pm SD) } & F value and sig. \\
\cline { 2 - 5 } Parameter & Non-diabetics & Prediabetics & Diabetics & $\begin{array}{l}\text { Diabetes } \\
\text { hypertension }\end{array}$ & with \\
\hline FBS & $82.37 \pm 11.01$ & $108.50 \pm 16.36$ & $170.60 \pm 35.87$ & $245.89 \pm 54.15$ & $185.9^{* *}$ \\
\hline RBS & $78.69 \pm 8.45$ & $95.40 \pm 3.71$ & $178.06 \pm 31.99$ & $237.93 \pm 35.28$ & $195.8^{* *}$ \\
\hline HBA1c & $4.83 \pm 0.47$ & $5.96 \pm 0.20$ & $8.31 \pm 1.52$ & $10.32 \pm 1.82$ & $156.3^{* *}$ \\
\hline TC & $161.22 \pm 19.54$ & $166.75 \pm 17.93$ & $173.24 \pm 22.66$ & $244.70 \pm 42.84$ & $232.1^{* *}$ \\
\hline TG & $103.59 \pm 23.94$ & $132.64 \pm 52.01$ & $172.36 \pm 17.73$ & $230.92 \pm 136.31$ & $26.6^{* *}$ \\
\hline HDL & $46.87 \pm 4.76$ & $39.33 \pm 2.74$ & $37.06 \pm 4.62$ & $32.89 \pm 2.49$ & $111.74^{* *}$ \\
\hline LDL & $100.15 \pm 19.93$ & $103.67 \pm 28.58$ & $106.64 \pm 19.75$ & $161.53 \pm 81.02$ & $20.1^{* *}$
\end{tabular}




\begin{tabular}{lccccc}
\hline VLDL & $25.28 \pm 4.72$ & $29.92 \pm 8.67$ & $40.46 \pm 4.27$ & $46.48 \pm 27.40$ & $20.20^{* *}$ \\
\hline TC:HDL & $3.44 \pm 0.15: 1$ & $4.25 \pm 0.48: 1$ & $4.69 \pm 0.48: 1$ & $7.54 \pm 1.73: 1$ & $172.13^{* *}$ \\
\hline LDL:HDL & $2.14 \pm 0.37: 1$ & $2.63 \pm 0.70: 1$ & $2.89 \pm 0.46: 1$ & $5.00 \pm 2.78: 1$ & $34.99^{* *}$ \\
\hline
\end{tabular}

${ }^{*}$ Significant difference as compared with all the groups (Significance at level $p<0.05$ ). ${ }^{* *}$ Highly significant (Significance at level $p<0.01$ ).

Correlation (denoted as ' $r$ ') is used to measure the strength of association between two variables and ranges between -1 (perfect negative correlation) to 1 (perfect positive correlation). It is interpreted as the absolute value of the correlation, considering any value equal to or more than 0.5 as strong, 0.3 to 0.5 as moderate, 0.1 to 0.3 as low and less than 0.1 as a negligible relationship. Tables 2-5 represent correlations among all the study variables to study the correlations between HbA1c, FBS and the total lipid profiles among non-diabetics, prediabetics, diabetics and diabetics with hypertension. HbA1c was moderately significantly correlated with FBS ( $r=0.49, p<0.01)$ and RBS $(r=0.39, p<0.05)$ in non-diabetics, strongly significantly correlated with FBS $(r=0.76, p<0.01)$ and RBS $(r=0.80, p<0.01)$ in case of diabetics. In case of prediabetics, there was a strong significant negative correlation of HBA1C with HDL $(r=-0.66$, $\mathrm{p}$ <0.05). Within diabetics with hypertension, HbA1c was significantly correlated with all the lipid components at varying levels. There was a strong significant correlation between $\mathrm{HbA1c}$ with FBS ( $\mathrm{r}=0.97, \mathrm{p}<0.01)$, TC:HDL $(\mathrm{r}=0.83, \mathrm{p}<0.01)$, TC $(\mathrm{r}=0.72, \mathrm{p}<0.01)$, moderate significant correlation with RBS $(\mathrm{r}=0.42, \mathrm{p}<0.01)$, VLDL $(\mathrm{r}=0.31 \mathrm{p}<0.05), \operatorname{LDL}(\mathrm{r}=0.38, \mathrm{p}<0.01)$ and LDL:HDL ( $\mathrm{r}=0.46, \mathrm{p}<0.01)$, significant low correlation with TG $(r=0.29, p<0.05)$ and negative strong significant correlation with HDL ( $r=0.89, \mathrm{p}<0.01)$.

Table 2: Correlation table between lipid profiles and HBA1c of non-diabetics.

\begin{tabular}{|c|c|c|c|c|c|c|c|c|c|}
\hline Parameter & FBS & RBS & HBA1C & $\mathrm{TC}$ & TG & HDL & LDL & VLDL & TC:HDL \\
\hline FBS & 1 & & & & & & & & \\
\hline RBS & $0.99^{* *}$ & 1 & & & & & & & \\
\hline HBA1C & $0.49^{* *}$ & $0.39^{*}$ & 1 & & & & & & \\
\hline TC & -0.02 & -0.24 & -0.04 & 1 & & & & & \\
\hline TG & $0.28^{*}$ & $0.41^{*}$ & 0.25 & 0.2 & 1 & & & & \\
\hline HDL & -0.09 & -0.23 & -0.05 & $0.95^{* *}$ & 0.2 & 1 & & & \\
\hline LDL & 0.17 & 0.31 & 0.11 & $0.59^{* *}$ & $0.66^{* *}$ & 0.27 & 1 & & \\
\hline VLDL & 0.22 & $0.41^{*}$ & 0.23 & 0.11 & $0.94^{* *}$ & 0.09 & $0.48^{* *}$ & 1 & \\
\hline Ch: HDL & 0.2 & -0.18 & 0.06 & $0.67^{* *}$ & 0.11 & $0.43^{* *}$ & $0.41^{* *}$ & 0.09 & 1 \\
\hline LDL: HDL & 0.26 & $0.49^{* *}$ & 0.16 & 0.19 & $0.66^{* *}$ & 0.14 & $0.88^{* *}$ & $0.50^{* *}$ & $0.27^{*}$ \\
\hline
\end{tabular}

Table 3: Correlation table between lipid profile and HBA1c of prediabetics.

\begin{tabular}{lcccccccc}
\hline \multicolumn{1}{c}{ Parameter } & FBS & RBS & HBA1C & TC & TG & HDL & LDL & VLDL \\
\hline FBS & 1 & & & & TC: HDL \\
\hline RBS & $0.99^{* *}$ & 1 & & & & \\
\hline HBA1C & 0.45 & $0.93^{*}$ & 1 & & & \\
\hline
\end{tabular}




\begin{tabular}{|c|c|c|c|c|c|c|c|c|c|}
\hline TC & 0.16 & 0.52 & -0.29 & 1 & & & & & \\
\hline TG & -0.45 & 0.21 & 0.05 & -0.46 & 1 & & & & \\
\hline $\mathrm{HDL}$ & -0.18 & -0.49 & $-0.66^{*}$ & 0.31 & -0.52 & 1 & & & \\
\hline LDL & -0.13 & 0.02 & -0.32 & 0.25 & -0.52 & 0.38 & 1 & & \\
\hline VLDL & -0.49 & 0.1 & -0.11 & -0.44 & $0.99^{* *}$ & -0.49 & 0.01 & 1 & \\
\hline TC: HDL & 0.31 & $0.95^{*}$ & 0.2 & $0.77^{* *}$ & -0.13 & -0.37 & -0.02 & -0.15 & 1 \\
\hline LDL: HDL & -0.09 & 0.17 & -0.16 & 0.18 & 0.07 & 0.17 & $0.97^{* *}$ & 0.11 & 0.06 \\
\hline
\end{tabular}

${ }^{*} \mathrm{p}<0.05,{ }^{* *} \mathrm{p}<0.01$

Table 4: Correlation table between lipid profile and HBA1c of diabetics.

\begin{tabular}{|c|c|c|c|c|c|c|c|c|c|}
\hline Parameter & FBS & RBS & HBA1C & $\mathrm{TC}$ & TG & HDL & LDL & VLDL & TC: HDL \\
\hline FBS & 1 & & & & & & & & \\
\hline RBS & $0.86^{* *}$ & 1 & & & & & & & \\
\hline HBA1C & $0.76^{* *}$ & $0.80^{* *}$ & 1 & & & & & & \\
\hline TC & -0.04 & -0.08 & -0.07 & 1 & & & & & \\
\hline TG & 0.01 & 0.23 & 0.09 & 0.16 & 1 & & & & \\
\hline HDL & 0.11 & -0.01 & 0 & $0.70^{* *}$ & 0.08 & 1 & & & \\
\hline LDL & -0.09 & -0.14 & -0.1 & $0.95^{* *}$ & -0.07 & $0.55^{* *}$ & 1 & & \\
\hline VLDL & -0.01 & 0.17 & 0.06 & 0.21 & $0.97^{* *}$ & 0.1 & -0.02 & 1 & \\
\hline TC: HDL & -0.21 & -0.14 & -0.1 & $0.42^{* *}$ & 0.16 & $-0.34^{* *}$ & $0.54^{* *}$ & 0.2 & 1 \\
\hline LDL:HDL & -0.2 & -0.17 & -0.13 & $0.57^{* *}$ & -0.11 & -0.14 & $0.75^{* *}$ & -0.07 & $0.92^{* *}$ \\
\hline
\end{tabular}

Table 5: Correlation table between lipid profile and HBA1c of diabetics with hypertension.

\begin{tabular}{|c|c|c|c|c|c|c|c|c|c|}
\hline Parameter & FBS & RBS & HBA1C & $\mathrm{TC}$ & TG & HDL & LDL & VLDL & TC: HDL \\
\hline FBS & 1 & & & & & & & & \\
\hline RBS & $0.45^{* *}$ & 1 & & & & & & & \\
\hline HBA1C & $0.97^{* *}$ & $0.42^{* *}$ & 1 & & & & & & \\
\hline $\mathrm{TC}$ & $0.70^{* *}$ & $0.33^{*}$ & $0.72^{* *}$ & 1 & & & & & \\
\hline TG & $0.27^{*}$ & 0.11 & $0.29^{*}$ & $0.29^{*}$ & 1 & & & & \\
\hline HDL & $-0.87^{* *}$ & $-0.33^{*}$ & $-0.89^{* *}$ & $-0.62^{* *}$ & -0.23 & 1 & & & \\
\hline
\end{tabular}




\begin{tabular}{|c|c|c|c|c|c|c|c|c|c|}
\hline LDL & $0.36^{* *}$ & -0.1 & $0.38^{* *}$ & $0.38^{* *}$ & $0.56^{* *}$ & $0.33^{*}$ & 1 & & \\
\hline VLDL & $0.30^{*}$ & 0.09 & $0.31^{*}$ & $0.28^{*}$ & $0.98^{* *}$ & 0.27 & $0.55^{* *}$ & 1 & \\
\hline TC: HDL & $0.81^{* *}$ & $0.37^{* *}$ & $0.83^{* *}$ & $0.97^{* *}$ & $0.31^{*}$ & $-0.77^{* *}$ & $0.40^{* *}$ & $0.31^{*}$ & 1 \\
\hline LDL: HDL & $0.43^{* *}$ & -0.06 & $0.46^{* *}$ & $0.42^{* *}$ & $0.58^{* *}$ & $-0.42^{* *}$ & $0.99^{* *}$ & $0.57^{* *}$ & $0.46^{* *}$ \\
\hline
\end{tabular}

${ }^{*} \mathrm{p}<0.05,{ }^{* *} \mathrm{p}<0.01$

Table 6 displays the demographic factors, lifestyle habits and clinical characteristics of non-diabetics, prediabetics, diabetics with and without hypertension. The mean ages of the nondiabetic and prediabetic populations were $40.19 \pm 12.40$ and $50.67 \pm 15.13$ years, respectively. Age had a statistically significant association with diabetes with hypertension (Pearson's chi square $(12)=53.51, \mathrm{p}<0.01$ ). The onset of diabetes occurred at a mean of $53.58 \pm 10.67$ years and diabetes with hypertension occurred at a mean of $51.21 \pm 9.80$ years. Similarly, the relationship between gender and diabetes with hypertension was statistically significant, $\chi^{2}(2)=17.62, p<0.01$. The males were more susceptible to diabetes and hypertension. There was also a significant relationship between occupation and diabetic nephropathy, $\chi^{2}(18)=120.67, p<0.001$. Individuals doing desk jobs and drivers were more inclined to develop hypertension with diabetes, whereas most people with businesses had diabetes. There was no significant relationship between food habits and diabetes or hypertension, $\chi^{2}(2)=0.70, \quad p>0.05$. Alcohol consumption had a significant association with diabetics with hypertension $\left(\chi^{2}(2)=22.00, \quad \mathrm{p}<0.01\right)$. All individuals consuming alcohol had diabetes or diabetes with hypertension. Moreover, diabetes and hypertension were also associated with smoking $\left(\chi^{2}(2)=12.21, \mathrm{p}<0.01\right)$. All smokers were patients with diabetes associated with hypertension; however, non-smoking did not guarantee escape from diabetes or hypertension. As expected, levels of physical activity significantly correlated with diabetes and hypertension, $\chi^{2}(4)=92.27, p<0.001$. People with sedentary habits had a higher chance of developing diabetes and eventually diabetes with hypertension; however, active individuals did not show any diabetes with hypertension. There was a significant correlation between BMI and diabetes with hypertension, $\quad \chi^{2}(2)=42.44, \quad p<0.001$. Overweight individuals were more likely to have diabetes and hypertension. Nevertheless, individuals with normal weight were not immune to diabetes or hypertension. The average BMI among the study population varied from normal weight for non-diabetics $(21.28 \pm$ $\left.1.58 \mathrm{Kg} / \mathrm{m}^{2}\right)$, prediabetics $\left(22.94 \pm 1.84 \mathrm{Kg} / \mathrm{m}^{2}\right)$, diabetics $\left(24.32 \pm 1.61 \mathrm{Kg} / \mathrm{m}^{2}\right)$ to overweight for diabetics with hypertension $\left(24.87 \pm 1.33 \mathrm{Kg} / \mathrm{m}^{2}\right)$. Abdominal obesity also had a significant correlation with diabetic nephropathy $\left(\chi^{2}(2)=35.26\right.$, $\mathrm{p}<0.01)$. The majority of individuals with abdominal obesity were diabetics along with nephropathy, whereas non-diabetics mostly had no abdominal obesity.

Table 6: Demographic data and lifestyle habits in non-diabetics, prediabetics, diabetics without and with hypertension.

\begin{tabular}{|c|c|c|c|c|c|c|c|}
\hline \multirow{2}{*}{ Variables } & \multirow{2}{*}{ Group } & Non-diabetics & Prediabetics & Diabetics & $\begin{array}{l}\text { Diabetics with } \\
\text { hypertension }\end{array}$ & $\chi^{2}$ & $\mathrm{p}$ value \\
\hline & & \multicolumn{6}{|c|}{ Frequency (Percent) } \\
\hline \multirow{5}{*}{ Age (years) } & $21-31$ & $16(29.6 \%)$ & $1(8.3 \%)$ & $1(1.4 \%)$ & $1(1.8 \%)$ & \multirow{5}{*}{53.51} & \multirow{5}{*}{0} \\
\hline & $32-45$ & $21(38.9 \%)$ & $4(33.3 \%)$ & $17(23.6 \%)$ & $16(28.6 \%)$ & & \\
\hline & $46-59$ & $11(20.4 \%)$ & $5(41.7 \%)$ & $30(41.7 \%)$ & $26(46.4 \%)$ & & \\
\hline & $60-73$ & $6(11.1 \%)$ & $1(8.3 \%)$ & $23(31.9 \%)$ & $13(23.2 \%)$ & & \\
\hline & Older than 73 & $0(0 \%)$ & $1(8.3 \%)$ & $1(1.4 \%)$ & $0(0 \%)$ & & \\
\hline \multirow{2}{*}{ Gender } & Males & $15(27.8 \%)$ & $3(25.0 \%)$ & 37 (51.4\%) & $36(64.3 \%)$ & \multirow{3}{*}{17.62} & \multirow{3}{*}{0.001} \\
\hline & Females & $39(72.2 \%)$ & $9(75.0 \%)$ & $35(48.6 \%)$ & $20(35.7 \%)$ & & \\
\hline \multirow{2}{*}{ Occupation } & Desk job type & $0(0.0 \%)$ & $0(0.0 \%)$ & $7(9.7 \%)$ & 27 (50.9\%) & & \\
\hline & Homemaker & $18(34.6 \%)$ & $6(50.0 \%)$ & $29(40.3 \%)$ & $16(30.2 \%)$ & 120.67 & 0 \\
\hline
\end{tabular}




\begin{tabular}{|c|c|c|c|c|c|c|c|}
\hline & Retired & $1(1.9 \%)$ & $0(0.0 \%)$ & $5(6.9 \%)$ & $3(5.7 \%)$ & & \\
\hline & Business & $2(3.8 \%)$ & $0(0.0 \%)$ & $17(23.6 \%)$ & $0(0.0 \%)$ & & \\
\hline & Office & $24(46.2 \%)$ & $6(50.0 \%)$ & $9(12.5 \%)$ & $1(1.9 \%)$ & & \\
\hline & Nurse and hospital staff & $7(13.5 \%)$ & $0(0.0 \%)$ & $1(1.4 \%)$ & $1(1.9 \%)$ & & \\
\hline & Driver & $0(0.0 \%)$ & $0(0.0 \%)$ & $4(5.6 \%)$ & $5(9.4 \%)$ & & \\
\hline \multirow{2}{*}{ Food style } & Vegetarian & $21(39.6 \%)$ & $4(36.4 \%)$ & $29(40.8 \%)$ & $19(33.9 \%)$ & \multirow{2}{*}{0.7} & \multirow{2}{*}{0.87} \\
\hline & Non-vegetarian & $32(60.4 \%)$ & $7(63.6 \%)$ & $42(59.2 \%)$ & $37(66.1 \%)$ & & \\
\hline \multirow{2}{*}{$\begin{array}{l}\text { Alcohol } \\
\text { consumption }\end{array}$} & Yes & $0(0.0 \%)$ & $0(0.0 \%)$ & $3(4.2 \%)$ & $12(21.8 \%)$ & \multirow{2}{*}{22} & \multirow{2}{*}{0} \\
\hline & No & $54(100 \%)$ & $12(100 \%)$ & $69(95.8 \%)$ & $43(78.2 \%)$ & & \\
\hline \multirow{2}{*}{ Smoking } & Yes & $0(0.0 \%)$ & $0(0.0 \%)$ & $0(0.0 \%)$ & $13(24.1 \%)$ & \multirow{2}{*}{12.21} & \multirow{2}{*}{0.007} \\
\hline & No & $15(100 \%)$ & $4(100 \%)$ & $25(100 \%)$ & $41(75.9 \%)$ & & \\
\hline \multirow{3}{*}{$\begin{array}{l}\text { Physical } \\
\text { level }\end{array}$} & Sedentary & 10 (18.9\%) & $6(50.0 \%)$ & $52(72.2 \%)$ & $53(94.6 \%)$ & \multirow{3}{*}{92.27} & \multirow{3}{*}{0} \\
\hline & Moderately active & $10(18.9 \%)$ & $2(16.7 \%)$ & $15(20.8 \%)$ & $3(5.4 \%)$ & & \\
\hline & Active & $33(62.3 \%)$ & $4(33.3 \%)$ & $5(6.9 \%)$ & $0(0.0 \%)$ & & \\
\hline \multirow{3}{*}{ BMI } & Underweight & $1(1.9 \%)$ & $0(0.0 \%)$ & $0(0.0 \%)$ & $0(0.0 \%)$ & \multirow{3}{*}{42.44} & \multirow{3}{*}{0} \\
\hline & Normal & $51(96.2 \%)$ & $10(83.3 \%)$ & $38(52.8 \%)$ & $26(46.4 \%)$ & & \\
\hline & Overweight & $1(1.9 \%)$ & $2(16.7 \%)$ & $34(47.2 \%)$ & $30(53.6 \%)$ & & \\
\hline \multirow{2}{*}{$\begin{array}{l}\text { Abdominal } \\
\text { obesity }\end{array}$} & Yes & $0(0.0 \%)$ & $1(8.3 \%)$ & $23(31.9 \%)$ & 26 (47.3\%) & \multirow{2}{*}{35.26} & \multirow{2}{*}{0} \\
\hline & No & $54(100 \%)$ & $11(91.7 \%)$ & 49 (68.1\%) & $29(52.7 \%)$ & & \\
\hline
\end{tabular}

\section{DISCUSSION}

The key findings were that the correlation of glycosylated hemoglobin with FBS, RBS and lipid profile increased with onset of diabetes. The maximum correlation of $\mathrm{HbA1c}$ with lipid components was observed for diabetics with hypertension. For diabetics, there was no significant correlation between HbA1c and any lipid components; however, there was a strong significant correlation with FBS and RBS. This contradicts findings by Taliyan et al. [4] and Meenu et al. [6] for different Indian states (namely, UP and Gujarat respectively). Alam et al. [1] also reported significant correlations between all components of the lipid profile and glycosylated hemoglobin. Maharjan et al. [13], Cohen et al. [18] and Arab et al. [19] reported significant correlations between glycosylated hemoglobin and TG, TC, LDL and FBS and non-significant correlation with HDL. Babikr et al. [2] also reported correlations of HbA1c with LDL. Ju et al. [20] and Devkar et al. [21] reported highly significant correlations between HbA1c and FBS, similar to our study; however, Devkar et al. [21] also reported correlations with TC, TG, and LDL, contradictory to our observations. Moreover, our results are in accordance with those of studies of diabetics by Sheikhpour et al. [10], Satyanarayana et al. [11] and Sultania et al. [5]. In prediabetics, we observed a negative correlation of HDL with HbA1c. This was also observed by Ahuja et al. [22] in type I diabetics. It was also realized that RBS did not work as an indicator for prediabetes; however, FBS could identify prediabetics. Therefore, FBS can be helpful in preventive diagnosis, where prediabetics can control their diabetes through diet and exercise. This is contradictory to findings by Wang et al. [15], where the FBS was unable to differentiate patients with abnormal HbA1c. The differences in the results can be explained by differences in geography, race or ethnic considerations [14,15].

Typically, dyslipidemia is characterized by elevated levels of lipid profile components, including TG, TC, LDL and VLDL excepting HDL, which follows the reverse trend [1]. Our lipid profile results in diabetics with hypertension completely matched those of dyslipidemics. The levels of all the lipid profile components are above the desirable levels for diabetics with hypertension, showing the progression of the spread of the harmful effects of diabetes to various body parts. The significant difference in the populations observed for the lipid profile of 
our sample populations was in accordance with the results of diabetics in studies bys Sultania et al. [5] and Meenu et al. [6].

All the diabetics with hypertension had abnormal lipid profiles, similar to dyslipidemia patients. However, for diabetics, only TG, HDL, VLDL, TC:HDL concord with findings in dyslipidemic patients. The prediabetics showed abnormal HDL and TC:HDL levels. There was a high degree of correlation between lipid profiles and glycosylated hemoglobin, especially for diabetics with hypertension. Therefore, for diabetics, it should be mandatory to test the levels of various components of the lipid profile at regular intervals, because they fall in the highrisk category for developing both dyslipidemia and hypertension. However, for non-diabetics and prediabetics, it is not a dependable marker for detecting future diabetic dyslipidemia or hypertension. Our study also showed that HbA1c cannot be used as a marker for dyslipidemia, in agreement with Sultania et al. [5]. Moreover, FBS can still be considered a precautionary parameter for the estimation of blood glucose in prospective diabetic individuals, whereas RBS may not be able to identify prediabetics. The risk factors for diabetics with hypertension were also identified in our study.

One limitation of this study is its cross-sectional nature. Our study area covered only one hospital in Bengaluru, Karnataka with a limited sample size of 191 individuals. Nevertheless, we believe hospital caters to a random study population, representing the true population of Karnataka and South India.

\section{CONCLUSION}

In conclusion, an overweight diabetic male with an inadequate and poor glycemic control, over the age of 46 years, with a desk job, leading a sedentary lifestyle and with an abdominal obesity has a higher chance of developing diabetes with hypertension. Further, habits like alcohol consumption and smoking adds to the risk. Lifestyle interventions like increased physical activity, weight control, consumption of a healthy diet, moderate intake of alcohol and smoking cessation will help to control the glycemic and lipid parameters in these patients.

\section{COMPLIANCE WITH ETHICAL STANDARDS}

\section{Ethics approval}

The study protocol was approved by the hospital ethics committee. No animals were used in this study. The authors have no ethical conflicts to disclose. All methods were followed according to the ethical standards of the responsible committee on human experimentation (institutional and national).

\section{Statement of informed consent}

All subjects signed informed consent forms to be included in the study.

\section{CONFLICT OF INTEREST}

The authors have no conflicts of interest to declare.

\section{ACKNOWLEDGEMENTS}

The authors thank Sri Krishna Sevashrama Hospital, Bengaluru for giving us an opportunity to conduct this study. Special thanks to the Nursing Superintendent, Mrs. Judith Fernandes for her help in recording patient lifestyle habits and BMI.

\section{FUNDING ORGANIZATIONS}

This research received no specific grant from any funding agency in the public, commercial or nonprofit sectors.

\section{REFERENCES}

1. Alam R, Verma MK, Verma P. Glycated hemoglobin as a dual biomarker in type 2 diabetes mellitus predicting glycemic control and dyslipidemia risk. Int J Life Sci. 2015;189:164-189.

2. Babikr WG, Alshahrani AS, Hamid HG, Abdelraheem AH, Shalayel MH. The correlation of HbA1c with body mass index and HDL-cholesterol in type 2 diabetic patients. Biomed Res. 2016;27:1280-1283.

3. Vinod Mahato R, Gyawali P, Raut PP, Regmi P, Singh KP, Pandeya DP, et al. Association between glycaemic control and serum lipid profile in type 2 diabetic patients: glycated haemoglobin as a dual biomarker. Biomed Res. 2011;22:375-380.

4. Taliyan S, Nagtilak S, Parashar P, Rastogi A. Correlation between Glycated hemoglobin and Lipid profile in Type 2 Diabetic population of district Meerut, UP. Int J Biomed Adv Res. 2016;7:534-536.

5. Sultania S, Thakur D, Kulshreshtha M. Study of Lipid Profile in Type 2 Diabetes Mellitus Patients and its Correlation with HbA1c. Int J Contemp Med Res. 2017;4: 2454-7379.

6. Meenu J, Jadeja Jayendrasinh M, Neeta M. Correlation between HbA1c values and lipid profile in type 2 diabetes mellitus. Int J Basic Appl Physiol. 2013;147:35-40.

7. Anand. Significance of Hbalc and lipid profile test in diagnosis and prognosis of diabetic and cardiovascular patients. Int J Med Health Res. 2017;3:105-109.

8. Hussain A, Ali I, Ijaz M, Rahim A. Correlation between hemoglobin $\mathrm{A} 1 \mathrm{C}$ and serum lipid profile in Afghani patients with type 2 diabetes: hemoglobin A1C prognosticates dyslipidemia. Ther Adv Endocrinol Metab. 2017;8:51-57.

9. Satyanarayana N, Kumar IU, Mudda SKSG, Kumar T. A study of correlation of BMI and lipid profile in Type 2 diabetes mellitus subjects at a tertiary care hospital. J Ev Med Den Sci. 2014;3:422-434.

10. Sheikhpour R, Sadeghian F, Rajabi S. Correlation between glycated hemoglobin, serum glucose and serum lipid levels in type 2 diabetes. IRST. 2013;5:12-15.

11. Senthilkumar N, Anandhasayanam A, Senthilvelu A, Rasheed M. Correlation observation Between HbA1c Values and Lipid Profile in Type II Diabetes Mellitus out patients. IJPRR. 2016;5:9-20.

12. Khan HA, Sobki SH, Khan SA. Association between glycaemic control and serum lipids profile in type 2 diabetic patients: HbA1c predicts dyslipidaemia. Clin Exp Med. 2007;7:24-29.

13. Maharjan P, Pandeya D, Joshi G, Hona S, Bhatta B, Hamza A, et al. Glycated Hemoglobin (Hba1C) Is a Predictor of Dyslipidemia in Type 2 Diabetes Nepalese Patients. Int J Adv Res (Indore). 2017;5:113-121.

14. Cohen RM, Smith EP, Arbabi S, Quinn CT, Franco RS. Do Red Blood Cell Indices Explain Racial Differences in the Relationship between Hemoglobin A1c and Blood Glucose? J Pediatr. 2016;176:7-9. 
15. Wang A, Shi Q, He JC, Uribarri J, York N, College M. Comparison of Sensitivity of HbA1c with Fasting Blood Glucose for Diagnosing Prediabetes in Chinese Americans. Curr Res Diabetes Obes J. 2018;5:1-6.

16. Snieder H, Sawtell PA, Ross L, Walker J, Spector TD, Leslie RDG. $\mathrm{HbA} 1 \mathrm{c}$ levels are genetically determined even in type 1 diabetes: evidence from healthy and diabetic twins. Diabetes. 2011;50:2858-2863.

17. Patel V, Shastri M, Gaur N, Jinwala P, Kadam AY. A study in prevalence of diabetic nephropathy in recently detected cases of type 2 diabetes mellitus as evidenced by altered creatinine clearance, urinary albumin and serum creatinine, with special emphasis on hypertension, hypercholesterolemia and obesity. Int J Ad Med. 2018;5:351-355.

18. Cohen J. Statistical power analysis for the behavioural sciences. Taylor \& Francis, Routledge. 1988.
19. Arab GA, Zahedi M, Kazemi V, Sanagoo A, Azimi M. Correlation between Hemoglobin A1C and Serum Lipid Profile in Type 2 Diabetic Patients Referred to the Diabetes Clinic in Gorgan. Iran J Clin Basic Res. 2018;2:26-31.

20. Ju HB, Xu XM, Shu ZZ, Li LF, Chen ZJ. Blood glucose not hemoglobin influenced glycosylated hemoglobin in type 2 diabetes patients on plateau of China. Int $\mathrm{J}$ Diabetes Dev Ctries. 2015;35:197-200.

21. Devkar V, Desai P, Prajapati P, Rao S, Desai A. Correlation between glycated hemoglobin and dyslipidemia in patients with type 2 diabetes mellitus in a tertiary care hospital, Maharashtra, India. Int J Sci Study. 2016;4:121-124.

22. Ahuja A, Roopakala MS, CR WD, Reddy S, Kumar KP. Glycated hemoglobin, dyslipidemia and risk of atherosclerosis in type 1 diabetic patients. Int J Diabetes Dev Ctries. 2011;31:18-21. 